\title{
COHESIVE ZONE MODEL IDENTIFICATION WITH DCB TEST PARAMETER ESTIMATION SENSITIVITY
}

\author{
Racine LY $^{1,2}$, Julien JUMEL ${ }^{1}$, Martin E.R. SHANAHAN ${ }^{1}$ and Florian LAVELLE ${ }^{2}$ \\ ${ }^{1}$ University of Bordeaux, I2M, UMR 5295 \\ 351 Cours de la Libération, F-33400 Talence, France \\ e-mail: \{racine.ly, julien.jumel, martin.shanahan\}@u-bordeaux.fr \\ ${ }^{2}$ Centre National d'Etudes Spatiales, DLA \\ 52 Rue Jacques Hillairet, 75612 Paris, France \\ florian.lavelle@cnes.fr
}

Keywords: Cohesive zone model, DCB, interface bonding, identification, confidence interval

\begin{abstract}
Crack initiation and propagation conditions during a double cantilever beam test under constant rate loading are investigated. Elastic-plastic cohesive zone model is considered to represent the interface separation law. A finite difference model is derived to evaluate the applied force versus specimen opening evolution. Cohesive zone model parameters effect on energy release rate is investigated to evaluate the ability of measuring protocol based on $J$-integral approach for cohesive zone model identification. Confidence intervals of these estimated parameters are evaluated rigorously considering reasonable assumption on numerical simulation conditions regarding experimental one, with nonlinear optimization algorithm.
\end{abstract}




\section{INTRODUCTION}

Cohesive zone models are now extensively used for evaluating the failure conditions of interfaces in laminates or bonded joints. They allow evaluating the development of cohesive stresses along the crack propagation path and are relevant for analysis of both crack nucleation and propagation conditions under monotonous loading. More advanced models are now proposed to deal with fatigue, creep or ageing damage of the interface so that more complex loading configuration can be analysed for the structure itself. Today, these models have been studied extensively from a theoretical and numerical point of view and several contributions have been proposed to compare the influence of the chosen CZM on failure load prediction for example.

Indeed, the CZM are still generally identified rather than measured so that the interface separation law shape is still chosen an empirical manner based on expected global behaviour. Bilinear shape is chosen for brittle like behaviour and trapezoidal shape when ductile behaviour is expected. This approach is clearly not appropriate and some efforts have been devoted to the development of specialized experimental techniques for measuring a precise manner the interface separation law (viz. cohesive stress versus displacement jump across the interface). These characterization protocols make use of new specimen geometry or loading system or using standard specimens utilize new analysis techniques. In the following we will concentrate on such new development and focus on the identification of CZM under mode I loading configuration with the Double Cantilever Beam (DCB) test. The traditional technique for "measuring" interface separation law from DCB crack propagation experiment generally consists in identifying the parameters of the CZM using numerical fitting procedure using the global applied force versus opening displacement evolution. Such protocol generally results in poor sensitivity on the global shape of the interface separation but allows precise evaluation of the interface fracture energy. Recently several attempts have been made to implement the digital image correlation (DIC) technique for more precise evaluation of the CZM. The side of the specimen is observed to measure the surface displacement of both adherents in the vicinity of the crack tip. It is expected that more numerous experiment data will improve the global sensitivity of the CZM and crack tip position identification. More original and sophisticated protocols have also been proposed such as the $J(\theta)$ method which is based on clever derivation of the $\mathrm{J}$ integral and direct measurement of the crack tip opening displacement and specimen end rotation. With this protocol no assumption concerning the CZM shape is required and the $J(\theta)$ method effectively provide direct estimate of the interface separation law which can be implemented also under mixed mode condition. Similar evaluations have been also obtained by using the effective crack length method leading to a more straightforward evaluation of the CZM. Finally, the use of strain gages for monitoring the development of damage along an interface in bonded joint or laminate have been recommended for a long time since such measurement seems to provide easy implementation and high sensitivity to damage detection. However, only recently, a direct CZM reconstruction scheme from backface strain monitoring during crack propagation experimental have been proposed while classical CZM identification procedure are generally used. However, direct CZM reconstruction methods are generally limited to simple monotonous loading configurations, direct evaluation of interface separation law under stationary, fatigue or environmental coupling still appears difficult at this step.

However, some works have been conducted for cohesive zone model parameters sensitivity and identification. Alfano and Fugiuele [1] have compared experimental data from mode-I crack test of adhesively bonded joints, with those obtained by modeling adhesive layer with exponential intrinsic cohesive zone model, bi-linear and trapezoidal. A good agreement be- 
tween experiments and simulation has been obtained and show that energy release rate is the only influencing parameter during propagation phase. They also show that bi-linear and exponential laws well reproduce the primer phase behavior, while trapezoidal law overestimates peak force at which propagation appears. Although critical energy release rate and maximum cohesive laws stress are often seen as most important parameters towards model outputs, Waas and Gustafson [2] concluded with kriging method that, maximum cohesive law stress does not significantly influence model outputs for crack tests like double cantilever beam and end notched flexure type. In addition, several authors have proposed cohesive zone model identification methods. Two approaches are worth noting: the first known as direct which makes no assumptions about law's shape, the second called indirect, imposing law's shape whose parameters are calibrated by minimizing a cost function that measure metric between numerical and experimental observations. In the first case, Sorensen and Jacobsen [3] have adopted Li and Ward [4] approach to successfully measure cohesive zone law by deriving energy release rate $\mathrm{J}$ with respect to the CTOD. For the second approach, Valoroso and Sessa [5] identify a posteriori an exponential law whose parameters are re-calibrated compared to experimental data. It should be noted that formulation of cohesive zone model mentioned above is made such that damage is embedded in that it corresponds to stiffness variations. They are used for materials with elastic/elastic-plastic time independent behavior. Here we focus on such material type.

In this article the crack initiation and propagation during double cantilever beam test under monotonous loading is simulated considering elastic perfectly plastic interface separation law. The classical force versus opening displacement curves are evaluated and compared with a theoretically derived form. Additionally the specimen end rotation and crack tip opening displacement as required by the $J(\theta)$ technique is conducted too. The sensitivity of $J(\theta)$ experimental techniques to the CZM parameters is evaluated and reconstruction of the CZM with the various techniques is conducted.

\section{ELASTIC PLASTIC COHESIVE ZONE MODEL}

Cohesive zone model consist of an interface phenomenological law represented by a relationship between cohesive stress and displacements jump at the bonding line. First models were derived by Barenblatt [6] and Dudgale [7] in 60's and since then, many models were proposed for fatigue, ageing and complex loads. Here we focus on an elastic-plastic cohesive zone model used as interface law for initiation and crack propagation during a mode-I fracture between to substrates assembled by structural adhesive e.g. for the double cantilever beam test.

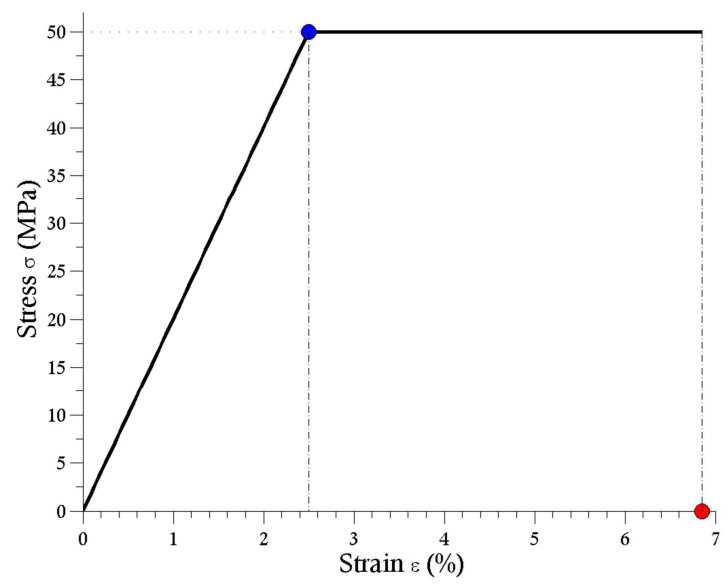

Figure 1 Elastic Perfectly Plastic cohesive zone model 
In figure 1 is illustrated a perfect elastic-plastic cohesive zone model. This model, as its name suggests, can be divided in two parts: elastic and plastic. The elastic part is characterized by two parameters $\sigma_{\max }$ and $\varepsilon_{e}$ linked by the initial stiffness $k_{a}$, and the second part depends on $\sigma_{\max }$ and strain at break $\varepsilon_{\max }$. According to strain partition principle, total strain can be written as the sum of an elastic reversible instantaneous part, and a second one where all dissipation processes are embedded. Here, we consider that this dissipation is provided by plastic phenomena. In addition, in order to take into account damage evolution, Kachanov damage evolution is used in term of mechanical property deterioration. Then, damage is represented here, by the secant stiffness decreasing at each load step. Concerning energy aspects, cohesive zone model formulations are made such that, the critical energy release rate $G_{c}$ is equal to the area under the law curve.

$$
G_{c}=\int_{0}^{\varepsilon_{\max }} \sigma(\varepsilon) \mathrm{d} \varepsilon
$$

In our case, the energy can be partitioned into elastic and plastic energy such that $G_{c}=$ $G_{e}+G_{p}$. In table 1 is represented quantities defined above and their formulation.

\section{DCB MODELING USING ELASTIC-PLASTIC COHESIVE LAW}

Double cantilever beam test is the common one to measure fracture energy in adhesively bonded joints. It sample consist of two adherents assembled by adhesive and loaded in order to initiate and propagate cracks. Standards like ASTM D5528 and ISO 25217, provides different data reduction techniques to compute fracture energy.

Parameters

$\mathrm{W}$

$\mathrm{t}_{\mathrm{a}}$

$\mathrm{E}_{\mathrm{a}}$

$\mathrm{k}_{\mathrm{a}}$

$G_{e}$

$\mathrm{G}_{\mathrm{p}}$

$\mathrm{G}_{\mathrm{c}}$
Formulation

$\begin{array}{cc}- & \text { Width } \\ - & \text { Thickness } \\ - & \text { Young modulus } \\ \frac{w E_{a}}{t_{a}} & \text { Initial stiffness } \\ \frac{1}{2} \sigma_{\max } \varepsilon_{e} & \text { Elastic energy } \\ \sigma_{\max }\left(\varepsilon_{\mathrm{m}}-\varepsilon_{\mathrm{e}}\right) & \text { Plastic energy } \\ \mathrm{G}_{\mathrm{e}}+\mathrm{G}_{\mathrm{p}} & \text { Critical energy }\end{array}$

Table 1 Parameters definition for elastic-plastic cohesive zone model

Many works have been proposed to model the double cantilever beam test with cohesive zone model as interface law and most of them use the finite element method. In this present, sample symmetry allows to consider only a half width and Winkler based modeling is used to derive constitutive equations. For this purpose, double cantilever beam sample is considered as beam lying on non-linear springs. 


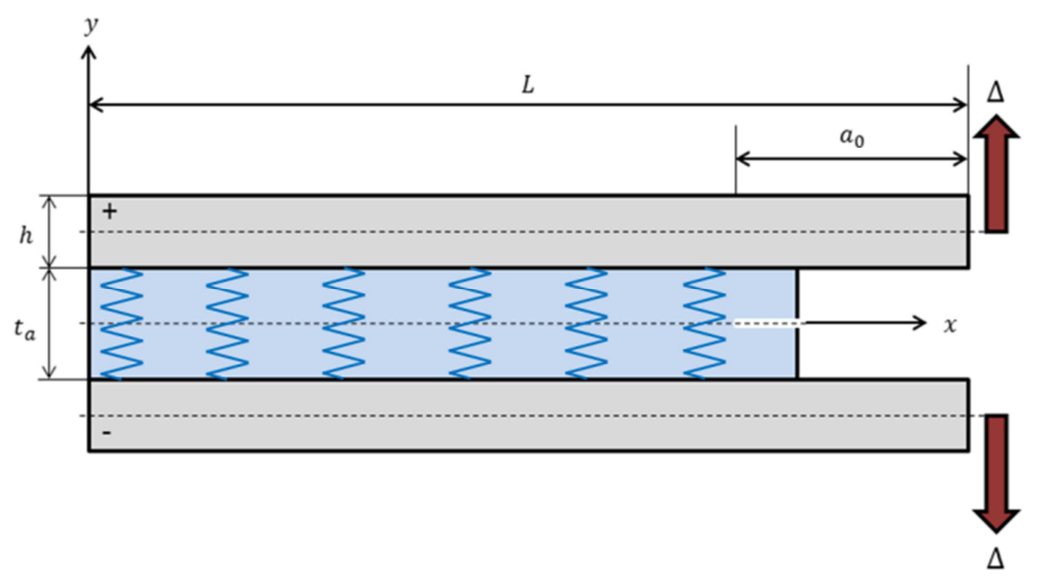

Figure 2 Double Cantilever Beam test specimen illustration

Timoshenko beam kinematics is used and springs follow a perfect elastic-plastic cohesive law. Then, cohesive stress can be seen as foundation reaction force due to opening load at the end-beam load point. In addition, the length of the bonded part is $L-a_{0}$ and the two slabs located at specimen right end are left unbounded over a distance equal to $a_{0}$ and considered as the initial crack length.

\subsection{Constitutive equations}

Specimen deformation is evaluated at each step of the experiment by solving local beam equilibrium and constitutive equations as presented below:

$$
\begin{gathered}
\frac{d M}{d x}+T=0 \\
\frac{d T}{d x}-w \sigma=0 \\
M=E I \frac{d \varphi}{d x} \\
T=\kappa G S\left[\frac{d v}{d x}-\varphi\right]
\end{gathered}
$$
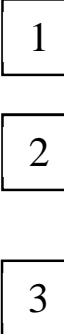

4

Where $M, T, \varphi$ and $v$ are bending moment, shear force, rotation and displacement respectively. Here $\sigma$ is the local stress defined by cohesive law presented in Section 2. Its expressions depend on local secant stiffness $\chi^{*}$ expand as below:

$$
\chi^{*}=\left\{\begin{array}{lc}
\frac{E_{a}}{t_{a}} & \text { if } \sigma<\sigma_{\max } \\
\frac{\sigma}{v} & \text { if } t_{a} \varepsilon \leq v \leq t_{a} \varepsilon_{\max } \\
0 & \text { if } v \geq t_{a} \varepsilon_{\max }
\end{array}\right.
$$


Combining equations (1) and (2) on one hand, and equations (2), (3) and (4) in the other, the driven equations for double cantilever beam test modeling obtained are expressed as follows :

$$
\begin{gathered}
\frac{d^{2} M}{d x^{2}}+w \chi^{*} v=0 \\
\frac{d^{2} v}{d x^{2}}-\frac{M}{E I}-\frac{w \chi^{*}}{\kappa G S} v=0
\end{gathered}
$$

\subsection{Numerical resolution and algorithmic aspects}

Normal displacements along beam and opening force at beam right end is conducted by solving equations (6) and (7) which are a PDE system with bending moment and displacements as unknown variables. $\chi^{*}$ is computed at each step using expressions and conditions in (5). Concerning resolution method, a finite difference method is used to approximate first and second derivatives: for first derivatives, a backward scheme is used and for second derivatives, a centered second order scheme. Then, at spatial coordinate $x_{k}$ and $x_{k+1}=x_{k}+\Delta x$, where $\Delta x$ is the spatial step one, can write:

$$
[M(x) \quad v(x)]=\left\{\begin{aligned}
{\left[\begin{array}{rr}
M_{k} & v_{k}
\end{array}\right] } & \text { if } x=x_{k} \\
{\left[\begin{array}{ll}
M_{k+1} & v_{k+1}
\end{array}\right] } & \text { if } x=x_{k}+\Delta x
\end{aligned}\right.
$$

Equations (6) and (7) can be rewritten as:

$$
\begin{gathered}
M_{k-1}-2 M_{k}+M_{k+1}+w d x^{2} \chi^{*} v_{k}=0 \\
-\frac{d^{2}}{E I} M_{k}-\left(2+\frac{w d x^{2} \chi^{*}}{\kappa G S}\right) v_{k}+v_{k-1}+v_{k+1}=0
\end{gathered}
$$

On can remark that (9) and (10) can be resume in a matrix system as follow:

$$
\left[\begin{array}{cccccc}
1 & 0 & -2 & \mathrm{wdx}^{2} \chi^{*} & 1 & 0 \\
0 & 1 & -\frac{\mathrm{dx}^{2}}{\mathrm{EI}} & -\left(2+\frac{\mathrm{wdx^{2 }} \chi^{*}}{\kappa G S}\right) & 0 & 1
\end{array}\right]\left[\begin{array}{c}
\mathrm{M}_{\mathrm{k}-1} \\
\mathrm{v}_{\mathrm{k}-1} \\
\mathrm{M}_{\mathrm{k}} \\
\mathrm{v}_{\mathrm{k}} \\
\mathrm{M}_{\mathrm{k}+1} \\
\mathrm{v}_{\mathrm{k}+1}
\end{array}\right]=\left[\begin{array}{l}
0 \\
0
\end{array}\right]
$$

Concerning boundary conditions at beam right side, an imposed displacement is applied and for beam left side, beam on elastic foundation conditions type is adopted. Defining a compliance matrix as $\mathrm{C}$, relation between normal displacements and rotation firstly, and shear force and bending moment in second, can be expressed according to degree of freedom allow in our configuration. Then, we obtain:

$$
\left[\begin{array}{c}
\mathrm{V} \\
\varphi
\end{array}\right]=\left[\begin{array}{cc}
\mathrm{C}_{\mathrm{vT}} & \mathrm{C}_{\mathrm{vT}} \\
\mathrm{C}_{\varphi \mathrm{T}} & \mathrm{C}_{\varphi \mathrm{M}}
\end{array}\right]\left[\begin{array}{c}
\mathrm{T} \\
\mathrm{M}
\end{array}\right]
$$


Below is illustrated the generic algorithmic scheme that was developed for double cantilever beam test using cohesive zone model as interface law. Three computations level appears depending on which part of the code is activated. Level 0 is related to raw data, level 1 to loop initiation, level 2 when the code operates inside the loop and finally level 3 when the stopping rule allows getting out the loop.

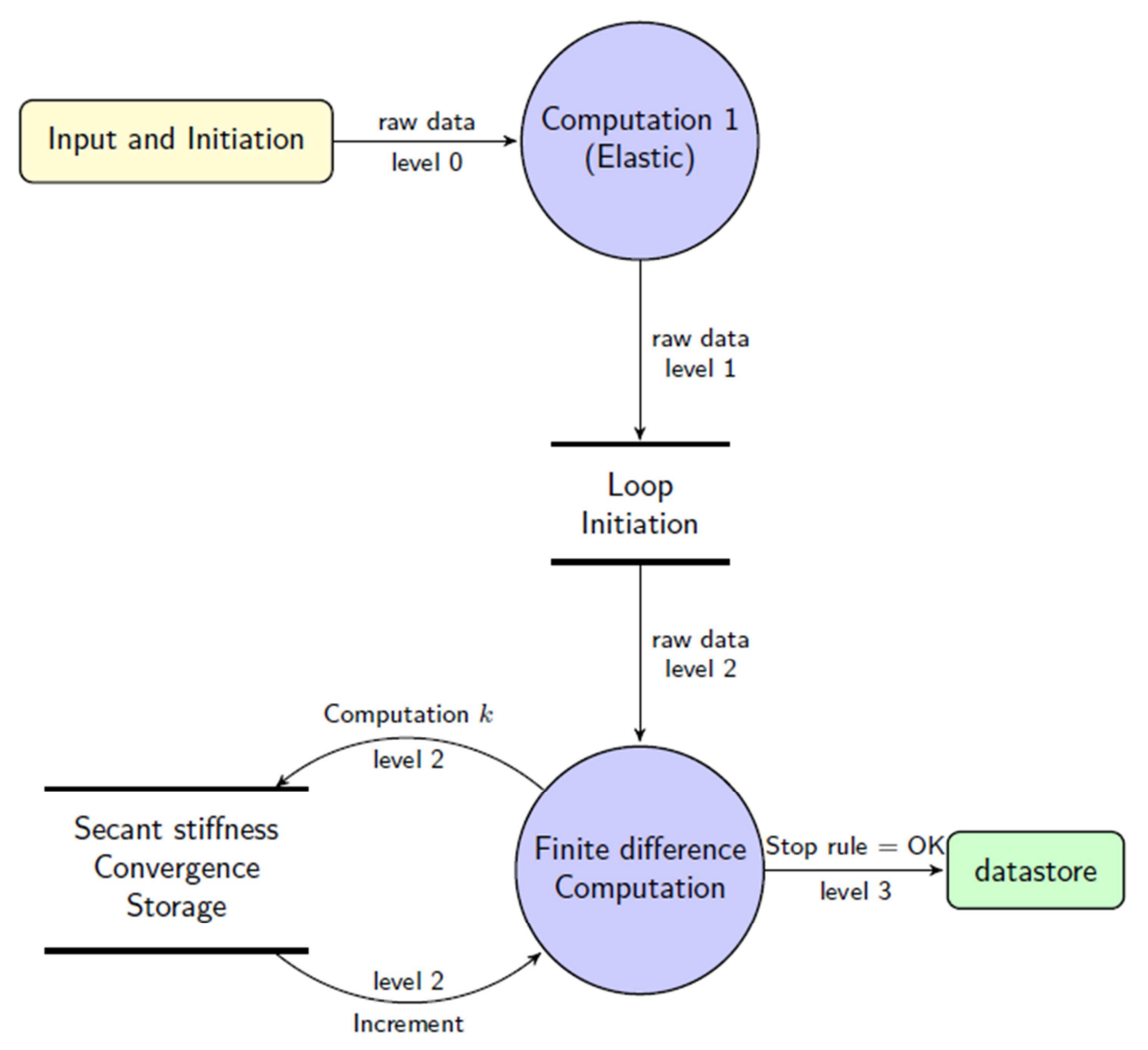

Figure 3 Algorithmic scheme for DCB experiment modeling

\section{NUMERICAL RESULTS}

In this section, are shown numerical results obtained from a double cantilever test simulation using an elastic perfectly plastic cohesive zone model as interfacial behavior for adhesive layer. We consider Aluminum substrate as Timoshenko beam with 70e9 Young modulus, 0.3 Poisson ratio and $200 \mathrm{~mm}$ length. In addition, a $30 \mathrm{~mm}$ initial crack length is defined and the specimen is $10 \mathrm{~mm}$ thick and $15 \mathrm{~mm}$ of width. For the cohesive zone model, three parameters are taken into account: Young modulus $\mathrm{E}_{\mathrm{a}}$, maximum local $\sigma_{\max }$ stress and strain at break $\varepsilon_{\text {max }}$. Initial values are respectively set to $2 \mathrm{GPa}, 50 \mathrm{MPa}$ and $6.85 \%$. Opening force $\mathrm{P}$ and opening displacement $\Delta$ at the specimen right end are computed, as energy release rate $\mathrm{J}$ using J-integral formula and apparent crack length. Finally, a constant rate displacement load is applied at specimen right end in order to charge it in Mode-I for initiation and crack propagation. Results are compared to analytical formula provided by single beam theory approach. 


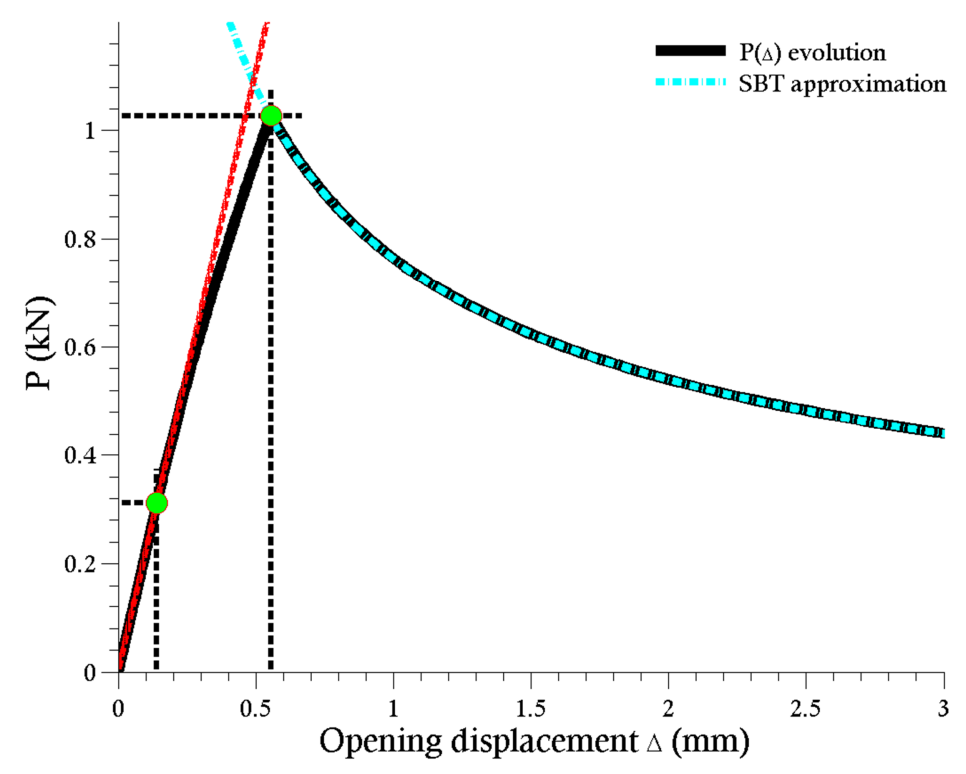

Figure 4 Opening force versus Opening displacement

In Figure 4 is shown the opening force according to the opening displacement load. The curve presents three different parts. The first one is linear and is referring to fast processes like those of elastic behavior and can be related to cohesive zone model elastic part. The second one is characterized by the loss of linearity and corresponds to the plastic part of the CZM. And the last part is related to the crack propagation. For this last, an analytical formula derived from single beam theory allow to relate $\mathrm{P}$ and $\Delta$ using simple beam theory (Eq. 11), the curve provided by this formula is illustrated by the cyan dotted lines and perfectly match our $\mathrm{P}(\Delta)$ crack propagation evolution.

$$
P=\left(\frac{4 E I}{9}\right)^{1 / 4}\left(w G_{c}\right)^{3 / 4} \frac{1}{\sqrt{\Delta}}
$$

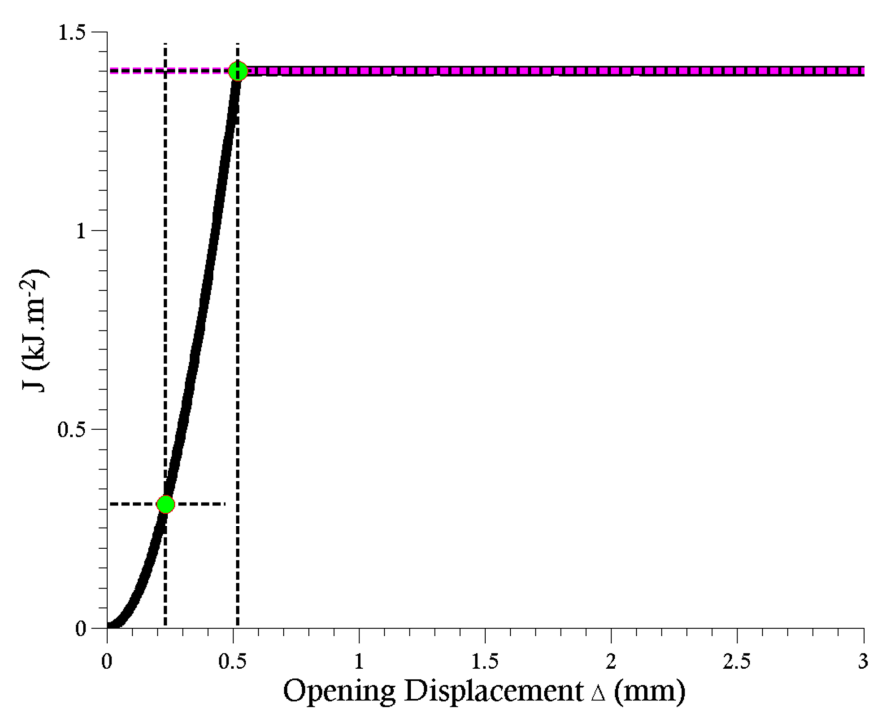

Figure 5 Energy release rate $\mathrm{J}$ according to Opening displacement 
Figure 5 shows three parts also, and they are related to same processes as for $\mathrm{P}(\Delta)$ evolution. However, a point has to be made concerning the propagation part. In fact, we assume in this paper a stationary propagation which induced a constant energy release rate during this phase and equal to the area under the cohesive zone model which should be an assumption made when loading speed is low. On can also remark that $\mathrm{J}$ becomes constant at $\Delta=0.5 \mathrm{~mm}$ and at this value in Figure 4, the force reached his maximum value $\mathrm{P}_{\max }=1.1 \mathrm{kN}$. Such facts that can be seen as crack propagation criteria. In Figure 6 is represented the same quantity $\mathrm{J}$ plotted in terms of crack opening displacement $\delta$, only crack initiation phase is represented because of during propagation phase, we assume that $\mathrm{J}$ is constant. According to $\mathrm{J}$-integral approach theory, $\mathrm{J}$ is written as path independent contour integral as:

$$
\mathrm{J}=\oint_{\Gamma} \sigma \cdot \mathrm{d} \delta
$$

Then, according to cohesive zone model shape, we can mention two essential parts of $\mathrm{J}$ $(\delta)$ evolution. In fact, the first part of CZM is the elastic one represented by an affine curve, then its integral should be a parabolic type curve, as we see in Figure 6 first phase. Using the same idea, CZM plastic is constant then its integral is a line as illustrated below. In conclusion, the modeling strategy used here by considering DCB specimen as Timoshenko beam lying on elastic perfectly plastic foundation through a Winkler type approach shows good agreement between analytical result provided by J-Integral approach and his computed evolution. The same accuracy can be found on crack propagation phase on Figure 4, where numerical results perfectly matched the theoretical evolution.

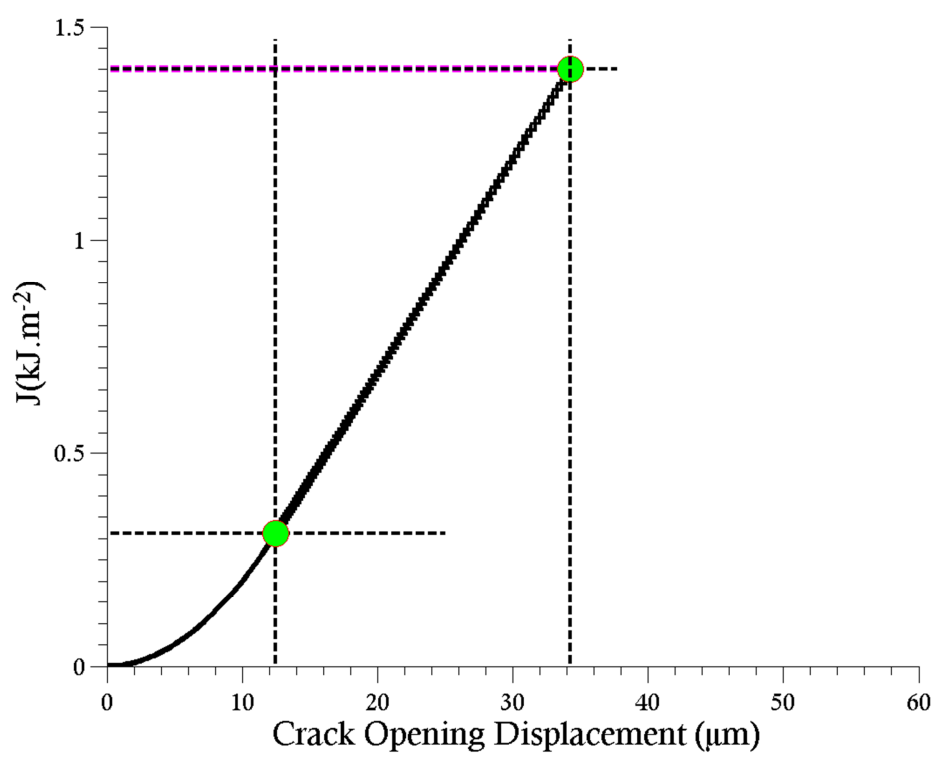

Figure 6 Energy release rate J according to Crack Opening Displacement 


\section{COHESIVE ZONE MODEL IDENTIFICATION}

\subsection{Sensitivity analysis}

Sensitivity analysis in order to quantify how CZM parameters affect $\mathrm{J}(\delta)$ evolution is conducted. For this, as influence indicator, we use gradient of $\mathbf{J}$ according to parameters and a weight factor is affected to each gradient in order to avoid units and scale gap as shown in Eq. 12. However, regarding to curve partitioning aspects discussed above, it's convenient to compute these quantities, first for the elastic part and second for the plastic phase. Such approach is chosen to facilitate cohesive zone model parameters identification, when coupling between parameters can be negligible or inexistent.
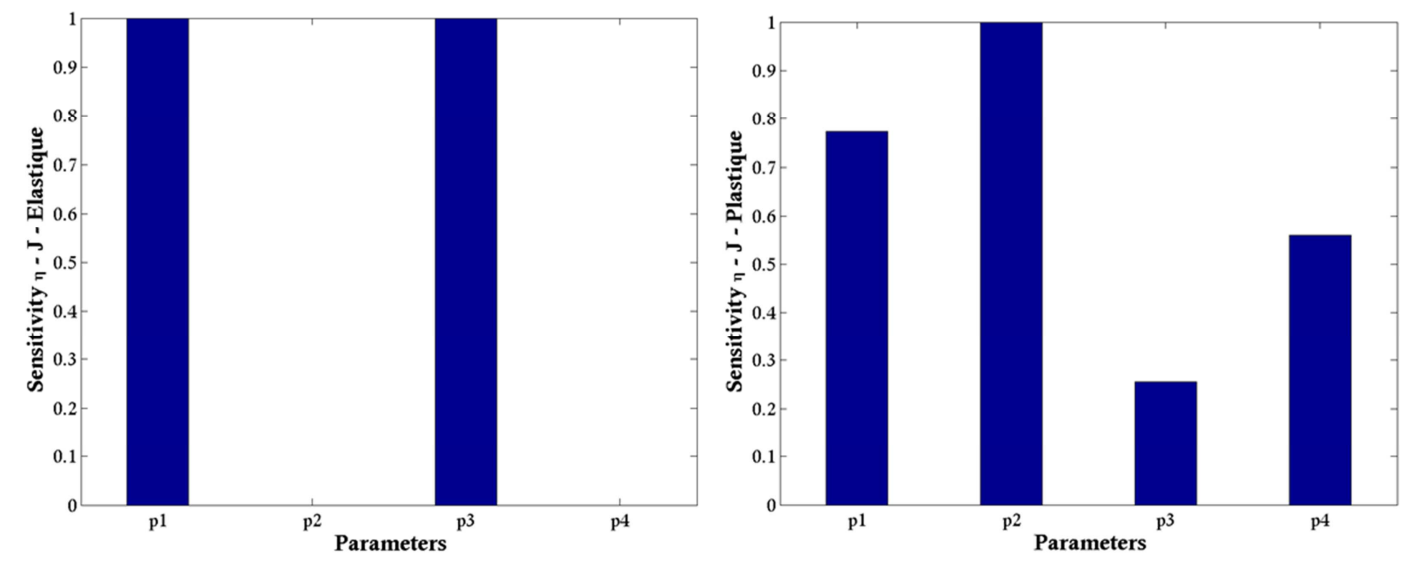

Figure 7 CZM parameters sensitivity index with $\mathbf{J}(\delta)$ evolution

$$
\eta_{\mathrm{i}}=\frac{\mathrm{p}_{0}}{\mathrm{~J}_{0}} \frac{\partial \mathrm{J}_{\mathrm{p}_{\mathrm{i}}}}{\partial \mathrm{p}_{\mathrm{i}}}
$$

Where, $p_{0}$ and $J_{0}$ are respectively parameters initial values and corresponding $\mathrm{J}$ values. Parameters p1, p2, p3 and p4 corresponds respectively to $t_{a}, \sigma_{\max }, E_{a}$, and $\varepsilon_{\max }$. We can see as shown in Figure 7 that adhesive thickness and Young modulus are the most important parameters in the elastic part. Nonetheless, we can also assume that adhesive thickness can be accurately measured and then his influence could be neglected. Young modulus is then the only driving parameter during the elastic phase as it could be expected. But sensitivity in plastic zone shows that $\mathrm{p} 2, \mathrm{p} 3$ and $\mathrm{p} 4$ have an influence on $\mathrm{J}$ response and a coupling between them should be taken into account. In this article, only p2 and p4 e.g. $\sigma_{\max }$ and $\varepsilon_{\max }$ taken separately are considered.

\subsection{CZM parameters confidence interval}

Cohesive zone model parameters identification is performed using minimization of a cost function with Levenberg-Marquardt algorithm in elastic and plastic part. Young modulus is identified in the first one and maximum stress and critical opening strain in the later. For this, synthetized experimental data are built by adding to simulated ones Gaussian noise with standard deviation of 10. In addition, random CZM parameters values are injected as seed for the model and asymptotic standard deviation is computed when convergence rule is satisfied. Parameters convergence and residuals evolution are provided in Figure 8, 9 and 10. Ratio between standard deviation and fitted parameters are reported in Table 2. 


$\begin{array}{cc}\text { Parameters } & \boldsymbol{\sigma}_{\mathbf{p}} / \mathbf{p}_{\text {fit }}(\mathbf{\%}) \\ \mathbf{E}_{\mathbf{a}} & 27.51 \\ \boldsymbol{\sigma}_{\max } & 8.86 \\ \mathcal{E}_{\max } & 8.93\end{array}$

Table 2 Parameters ratio: standard deviation obtained over fitted values
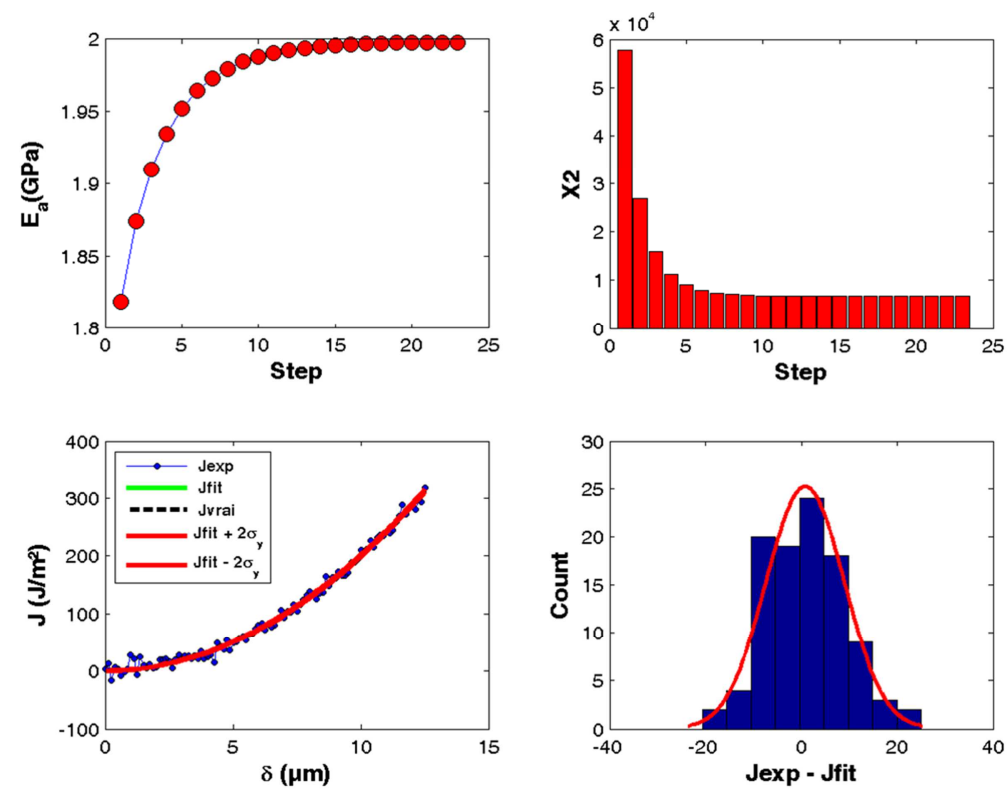

Figure 8 Young modulus identification
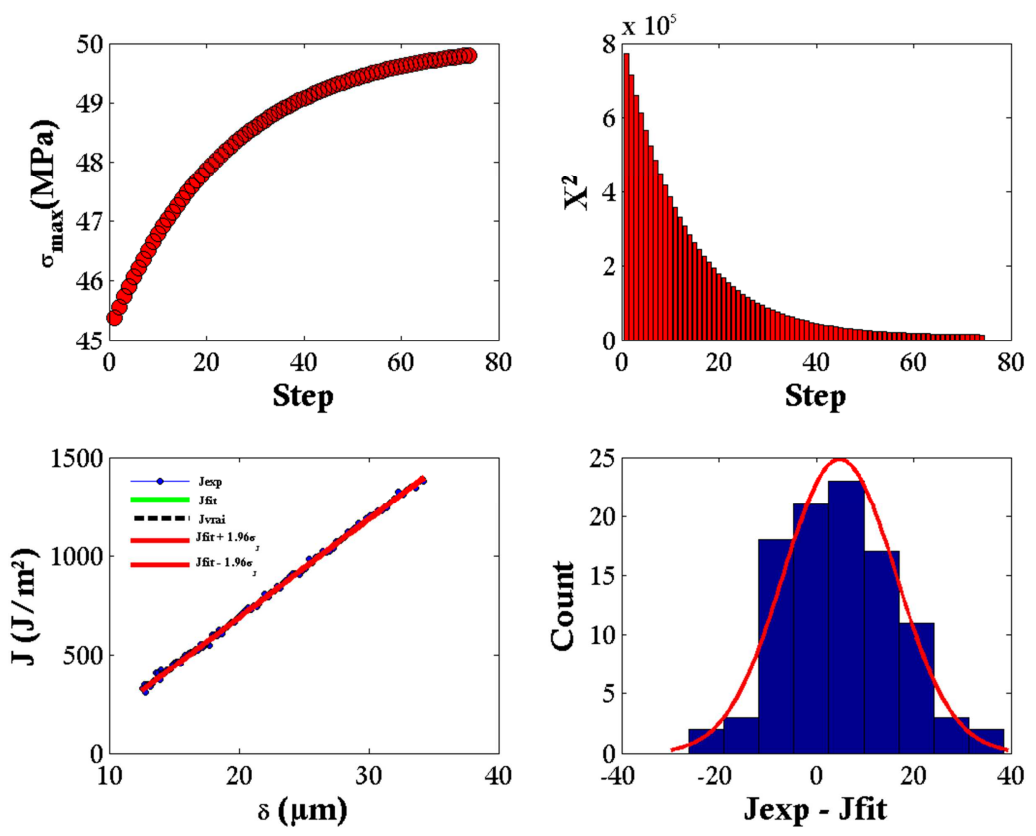

Figure 9 Maximum stress identification 

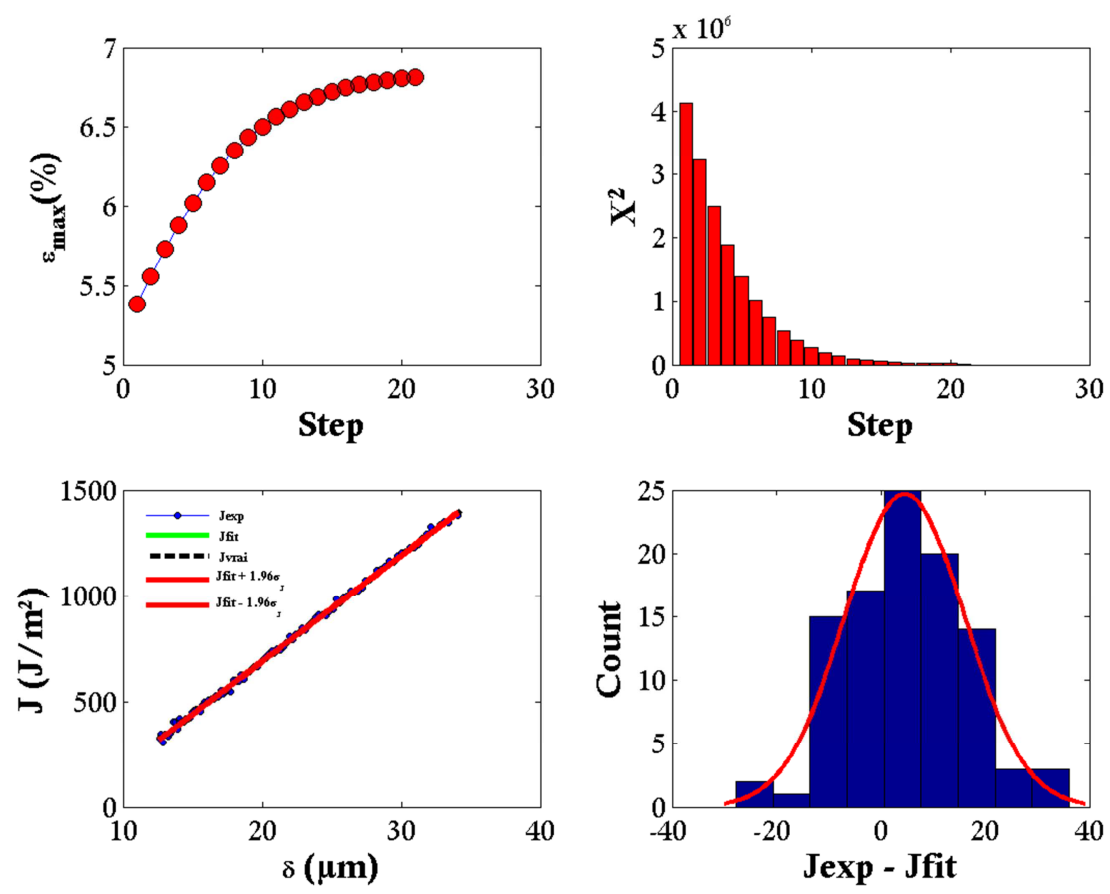

Figure 10 Strain at break stress identification

\section{CONCLUSIONS}

Crack initiation and propagation conditions during a double cantilever beam test under constant rate loading were investigated. Elastic-plastic cohesive zone model was considered to represent the interface separation law. A finite difference model was derived to evaluate the applied force versus specimen opening evolution and the parameters effect on energy release rate based on J-integral approach. Asymptotic standard deviation obtained from an optimization process, provide tight confidence interval. Since J could be measure with three parameters which is force, quantifiable with the traction machine and cross section rotation measurable with inclinometers, a cohesive zone model could be obtain using $\mathrm{J}$ first derivative according to crack opening displacement. The latter is also accessible from digital image correlation.

Future works must be devoted to more sophisticated sensitivity index estimation like those stochastic for example, and a comparison with experimental data in order to validate such model. 


\section{REFERENCES}

[1] M. Alfano and al, Mode I fracture of adhesive joints using tailored cohesive zone model. International Journal of Fracture, 157,193-204, 2009.

[2] A.M. Waas P.A. Gustafson. The influence of adhesive constitutive parameters in cohesive zone finite element models of adhesively bonded joints. International Journal of Solids and Structures, 46, 2201-2215, 2009.

[3] T.K. Jacobsen B.F. Sorensen. Determination of cohesive laws by the $\mathrm{j}$ integral approach. Engineering Fracture Mechanics, 70, 1841-1858, 2003.

[4] R.J. Ward V.C. Li. A novel testing technique for post-peak tensile behavior of cementitious materials. Fracture Toughness and Fracture Energy, 183-195, 2000.

[5] N. Valoroso and al. Identification of mode-i cohesive parameters for bonded interfaces based on DCB test. Engineering Fracture Mechanics, 104, 56-79, 2013.

[6] G.I. Barenblatt. The mathematical theory of equilibrium cracks formed in brittle fracture. Zhurnal prikladnoy mekhaniki i tekhnicheskoy fisiki, 4, 3-56, 2000.

[7] D.S. Dudgale. Yielding of steel sheets containing slits. Journal of the mechanics and physics solids, 8(2), 100-104, 1960. 